\title{
SOLAR ORBITER STRATEGIES FOR EMC CONTROL AND VERIFICATION
}

\author{
M. Pudney ${ }^{(1)}$, S. King ${ }^{(2)}$, T. Horbury ${ }^{(3)}$, M. Maksimovic ${ }^{(4)}$, C. J. Owen ${ }^{(5)}$, P. Laget $^{(6)}$ \\ (1)Airbus, Gunnels Wood Road, Stevenage, SG1 2AS, UK, Email:maxsim.pudney@airbus.com \\ (2) Airbus, Gunnels Wood Road, Stevenage, SG1 2AS, UK, Email: steve.t.king@airbus.com \\ (3) Imperial College London, South Kensington, London, SW7 2AZ, UK, Email: t.horbury@imperial.ac.uk \\ (4)LESIA, Meudon, 92195, Paris, FR,Email:milan.maksimovic@obspm.fr \\ (5) Mullard Space Science Laboratory, UCL, Holmbury St Mary, Dorking, RH5 6NT, UK, Email: c.owen@ucl.ac.uk \\ (6) ESA-ESTEC, Keplerlaan 1, 2201 AZ Noordwijk, NLD,Email:philippe.laget@esa.int
}

\begin{abstract}
Solar Orbiter, due for launch in February 2020, is an ESA mission to investigate how the Sun creates and controls the heliosphere. Solar Orbiter will combine insitu particle and field measurements with remote sensing observations of the Sun taken as close as 0.28AU (astronomical units). In order to make in-situ measurements, particularly at particle kinetic scales, it is necessary to establish and maintain control of the electro-static (ESC) and electro-magnetic compatibility $(\mathrm{EMC})$, between platform and instruments alike. We present the strategies employed in the ESC/EMC control of the Solar Orbiter mission, touching on the properties of key equipment such as the Solar Arrays, Reaction Wheels and Instrument Boom (I-Boom), and the proposed verification methodology for the system level EMC test campaign due to take place in 2019.
\end{abstract}

\section{MISSION}

Solar Orbiter is a European Space Agency (ESA) mission and the first medium sized craft of its Cosmic Vision 2015-2025 program (Fig. 1). The spacecraft is due for launch in February 2020. Solar Orbiter is a mission that aims to answer the question of how the Sun creates and controls the inner heliosphere. The primary means of influence is the solar wind: a tenuous outward flow of plasma, primarily composed of protons and electrons, originating from the Sun and extending into the outer solar system.

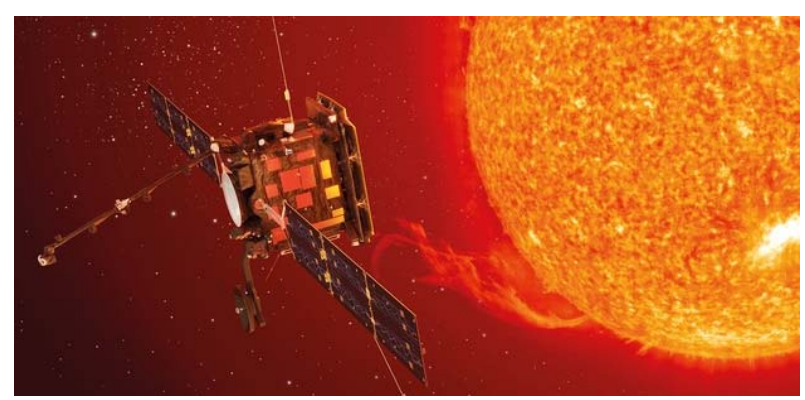

Figure 1. Artist's impression of Solar Orbiter [4].

Crucially, Solar Orbiter will make links between what it observes on the Sun (through remote sensing) and what it detects at the location of the spacecraft (through insitu measurements) in the inner heliosphere (Fig. 2).
The spacecraft orbit will allow periods of near corotation with the Sun. This provides the opportunity for extended observations of solar features. As the mission proceeds, the spacecraft will orbit with increased inclination out of the ecliptic plane so that it can perform observations of the polar regions of the Sun. Due to the necessary simultaneous operation of in-situ and remote sensing payloads (i.e. their synergy), EMC between payloads (and platform) is of paramount importance.

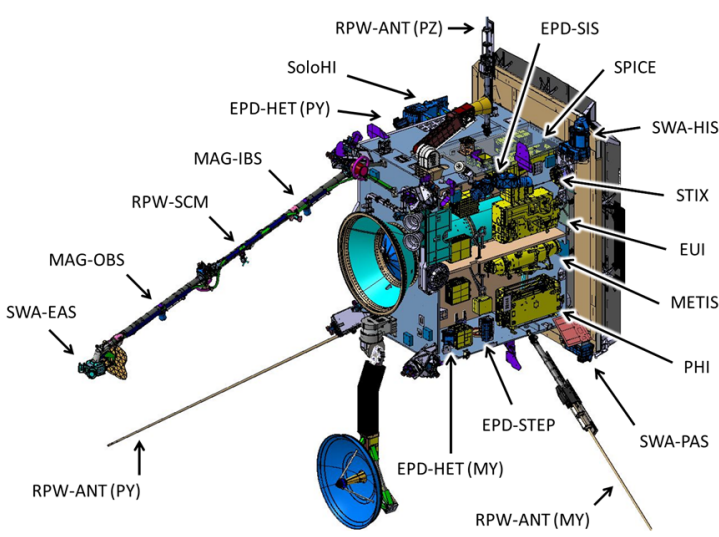

Figure 2. Solar Orbiter payload suite accommodation.

\section{NEED FOR MAGNETIC CLEANLINESS}

The in-situ payload includes three key instrument suites that are sensitive to the ESC/EMC environment of the spacecraft: SWA (Solar Wind Analyser - a suite of electron, proton/alpha-particle and heavy ion particle detectors), MAG (two sensors to measure the DC-64Hz magnetic field) and RPW (Radio Plasma Waves - a suite of three electric field antennas and a search coil magnetometer to measure the AC magnetic field). In order for each to achieve their respective science objectives, the platform contribution to the DC electric, AC electric, DC magnetic and AC magnetic fields must be carefully controlled, in some areas to an unprecedented precision. The driving science requirement is to observe the physics of the solar wind at kinetic scales, particularly those processes tied to electrons, which necessitates observations of $1 \mathrm{eV}$ low energy electrons, variations in DC magnetic field of order $10 \mathrm{pT}$ over time periods between 1 second and 1 hour, and for $\mathrm{kHz}$ frequencies $\mathrm{AC}$ electric fields as low as $1 \mathrm{nV} / \mathrm{m}$ and $\mathrm{AC}$ magnetic fields as low as $10 \mathrm{fT}$. 


\section{ESTABLISH REQUIREMENTS BASELINE}

Unlike missions such as Cassini [1] and Ulysses [2], Solar Orbiter is not able to employ a very long instrument boom (I-Boom), which is constrained to a length of approximately $4 \mathrm{~m}$. This is primarily due to the requirement to keep the boom in shadow behind its Sunfacing heatshield, and prevents dangerous solar exposure of the I-Boom instruments close to the Sun. As a result, ESC/EMC control and mitigation at unit level is even more important, and the establishment of an EMC Working Group (WG) combining specialists from Industry, ESA and the relevant Principal Investigators (PIs) at an early stage was beneficial.

The first task of the EMC WG was to translate the challenging science requirements into a measurable, meaningful set of technical requirements which could be practically specified and verified by industry. For example, one outcome was the translation of the acceptable spacecraft-induced deflection limits for electrons and protons reaching the sensors into a maximum integrated magnetic field along the path of any given particle within each sensor field-of-view (FOV), which can be calculated analytically from the magnetic budget (Fig. 3).

Example: deriving the
integrated magnetic
field requirement.
Top level: require
electrons to deflect $<1^{\circ}$
Charged particle in a
magnetic field experiences
Lorentz Force:
F $=\mathrm{q} \mathrm{v} \times \mathrm{B}=\mathrm{m}_{\mathrm{q}} \frac{\Delta \mathrm{v}}{\Delta \mathrm{t}}$
Small angle approximation:
sin $(\alpha)=\frac{\Delta \mathrm{v}}{\mathrm{v}}=\frac{\mathrm{qB} \Delta \mathrm{t}}{\mathrm{m}_{\mathrm{q}}}=\frac{\mathrm{q} \mathrm{B} \Delta \mathrm{x}}{\mathrm{m}_{\mathrm{q}} \mathrm{v}_{\mathrm{q}}}$

Figure 3. Derivation of the integrated magnetic field requirement for SWA-EAS (Electron Analyser System).
A second outcome was the derivation of a suitable maximum resistance requirement of $3 \mathrm{k} \Omega$ between an external surface and the spacecraft ground, based on the expected solar wind environment, the expected sunlit potential, the area of the Sun-facing heatshield, and the resulting incident and photo-emitted currents at $0.28 \mathrm{AU}$.

A third outcome was the derivation of enveloping AC electric and magnetic field emission requirements applicable to all unit suppliers (platform \& payload). Though instruments naturally test for self-emission of signals relevant to their own receivers, in order to demonstrate lack of self-contamination, this may not routinely happen for emission of signals sensitive to other instruments. These enveloping emission limits (Fig. 4), requiring unprecedented low levels of disturbance, needed to be repeatable and formally verifiable. This in turn required a significant extension from standard test practices, even after scaling for distance attenuation. Close co-ordination with specific test houses ensured accurate and repeatable sensitive measurements were possible, particularly with Prime and ESA EMC expert support on site during the unit level characterisation measurements.
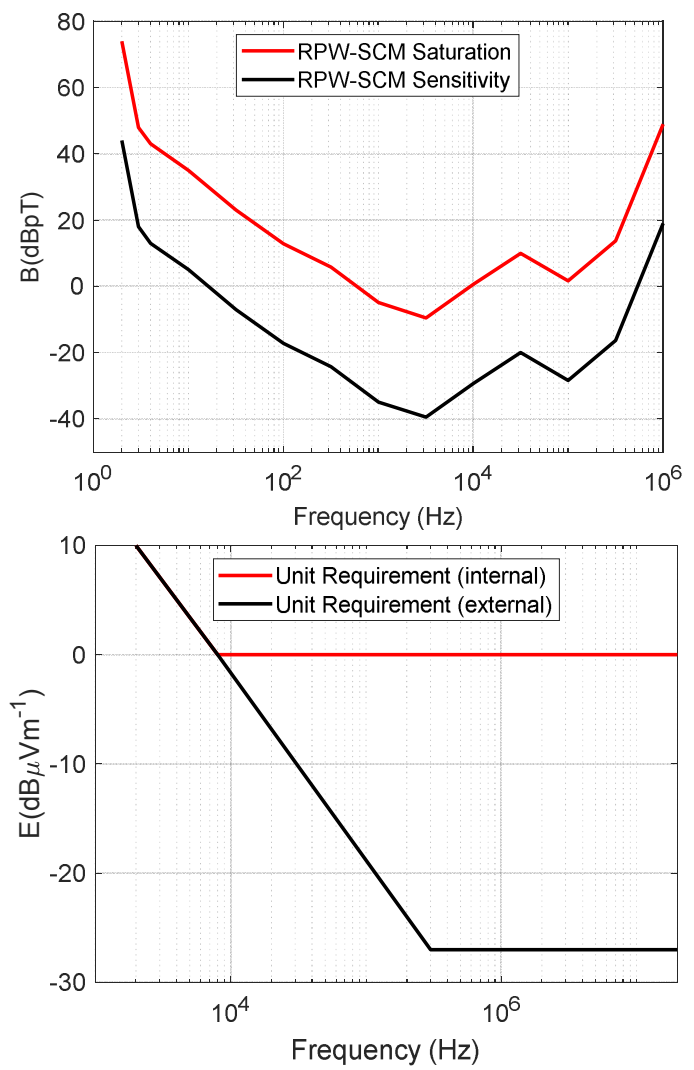

Figure 4. Derived unit characterisation levels for $R P W$ $S C M$ (top) and RPW-ANT (bottom). With respect to the $R P W-A N T$ figure, units internal to the $S / C$ have additional shielding and therefore the on-ground unit characterisation limit level is relaxed as shown. 
As an example, to verify the MAG variations requirement (to a precision of $5 \mathrm{pT}$ ) and RPW-SCM AC magnetic field requirement, units were tested at close distances and the results extrapolated to assess the impact at the in-flight positions. For the RPW-ANT AC electric field measurements, large monopoles had to be used, in conjunction with a pre-amp, fibre optic link, and FFT functionality in order to achieve the best possible noise floor on the ground (represented by the black line in Fig. 4 bottom). It was necessary to ensure the electrical ground support equipment (EGSE) was far from the test chamber and well-grounded / shielded in order to prevent interference in this very sensitive test.

Equally strong control over conducted emissions was imposed for early testing at board and Engineering Model (EM) level.

\section{EMC DESIGN AND CONTROL}

\subsection{Electric Field Control}

The key criterion for adequate electric field control is to limit external voltages from building up on the outside of the spacecraft to prevent adverse deflection of electrons (Fig. 5). Nominally this is achieved by grounding conductive outer MLI blankets to the structure, which on Solar Orbiter was chosen to be Black Kapton 160XC. Early analysis was performed following questionnaire responses from industrial subcontractors and instruments alike on any externally facing non-conductive materials, with deviations analysed on a case-by-case basis according to a process agreed with the Principal Investigators (PIs).
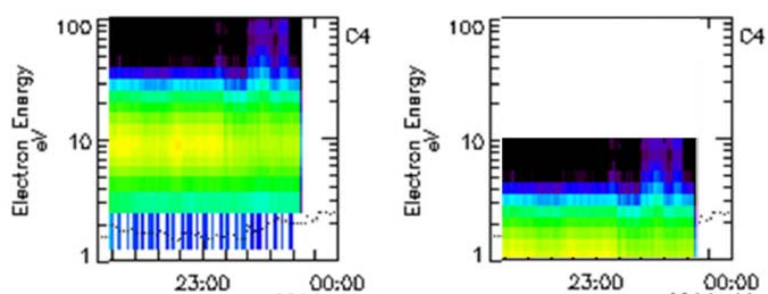

Figure 5. Example electron energy distribution with time (left) for a $\mathrm{S} / \mathrm{C}$ potential of $0 \mathrm{~V}$ (right) for a $\mathrm{S} / \mathrm{C}$ potential of $-10 \mathrm{~V}$ (where low energy electrons are slowed before reaching the sensor, or even repelled, leaving an incomplete distribution).

By performing measurements of the bulk conductivity of such materials, it was possible to more accurately model any space charge 'bubbles' that form in flight around unavoidable use of insulating materials. This modelling was done using the SPIS software [3], and to predict any impacts on the observed electron populations arriving at the key SWA-EAS instrument, which is positioned at the end of the boom. This led to a change in the Solar Array design to include an externally conductive single layer insulation (SLI) cover for the rear-surface carbon fibre panels, and revealed the limitation of standard radiator paint used for telecom applications when applied to science applications. Instead, for some applications such as the stood-off radiator assemblies (SORAs) and instrument boom hinges, the ENBIOC Solar White paint with ITO (indium tin oxide) was chosen, while ENBIO Solar Black was used for the HGA (high gain antenna), following successful verification of their bulk conductivities.

\subsection{Magnetic Field Control}

Each unit was assessed as part of its design review for any magnetic materials and a measurement performed to quantify the magnetic moment by determination of equivalent dipoles, which was then used to construct a system magnetic budget.
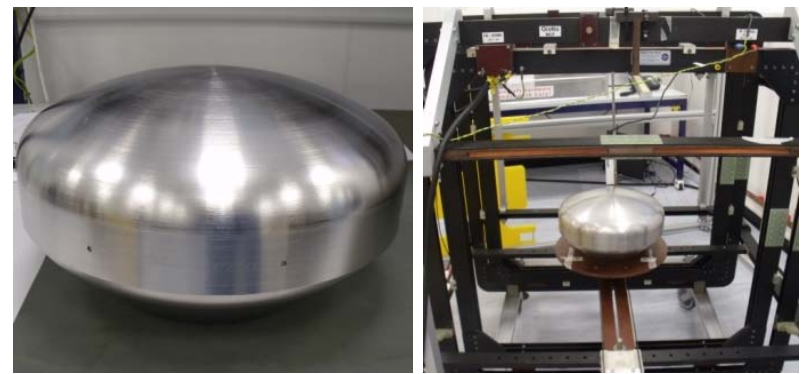

Figure 6. (left) Reaction wheel magnetic shield (right) tested on the Magnetic Coil Facility (MCF) Helmholtz coil in Airbus, Stevenage.

At Prime Contractor design level, the location of the worst-offending units was chosen to minimize the magnetic impact, for example by arranging the accommodation of latch valves and thrusters in compensating anti-parallel pairs. The vast majority of equipment was also de-magnetized at supplier level prior to delivery to Prime for installation. All heaters were specified to be self-compensating heater mats that minimise magnetic field generated due to stacked backwiring. In general, the use of steel in the design was avoided, or in cases of design necessity, de-magnetised.

Following assessment of measurement results, key interventions were made in a few areas. These include the replacement of the I-Boom hold-down release mechanism (HDRM) with a smaller, less magnetic, device, and the introduction of compensation magnets for thruster pairs to further attenuate the field (from $4 \mathrm{Am}^{2}$ for a single thruster to $<500 \mathrm{mAm}^{2}$ for a compensated thruster pair).

After arrival, the units were stored and integrated within a magnetically controlled access zone (CAZ) inside which only trained personnel were allowed to work, and tools were all magnetically screened prior to entry. 


\subsection{AC Electric and Magnetic Field Control}

Budget and schedule constraints, plus the heritage from BepiColombo, mandated the use of existing equipment wherever possible, which prohibited the use of crystal controlled oscillators and synchronised power converters for most platform units. As the AC electric and magnetic field requirements on Solar Orbiter are extremely stringent, a series of test campaigns at supplier level was performed early in the program, to de-risk the possibility of high emissions being found after flight unit build.

Where serious problems were identified some mitigation strategies were employed, such as:

- Additional electric field shielding (applied to the star tracker and communications module);

- Magnetic field shielding (used to attenuate the emissions from the reaction wheels) (Fig. 6);

- Frequency shifting (to move signals from payload AC heaters to a less sensitive RPW frequency band).

In general, the spacecraft uses aluminium face skins for its external structure housing, with apertures and slots taped with conductive cho-foil tape. The harness, which is externally wrapped in aluminium, is also taped regularly to the structure (approximately every $500 \mathrm{~mm}$ ), which has been demonstrated to reduce emissions significantly during unit EMC test campaigns.

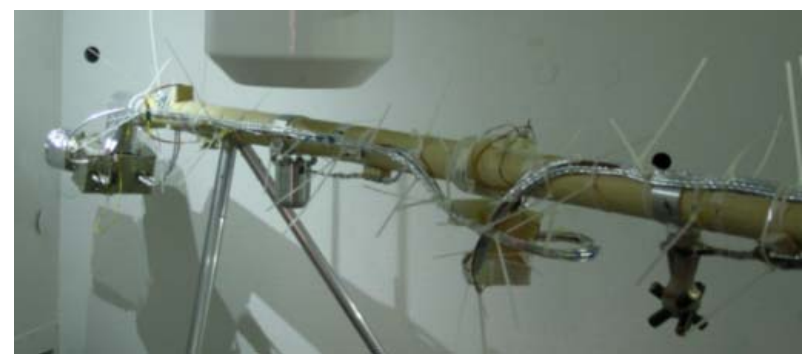

Figure 7. Instrument boom compatibility test at PTB.

To assist de-risking of the instrument boom payload inter-compatibility, a dedicated campaign took place at PTB Berlin (Physikalisch-Technische Bundesanstalt) in a nested mu-metal chamber that confirmed the acceptability of the baseline boom design and instrument accommodation (Fig. 7) [5]. The test facility used a superconducting quantum interference device (SQUID) to confirm instrument operation in an extremely low magnetic noise environment.

\section{EMC ANALYSIS}

\subsection{Magnetic Field Analysis}

The results from unit level testing were collated into a magnetic budget, which was then used to predict the DC magnetic field at the MAG locations, as well as the integrated fields observed in the particle sensor fields of view (e.g. SWA-EAS). For dipoles where the magnetic moment magnitude was known but not the direction, a random orientation was assigned, which could then be varied in a Monte Carlo sensitivity analysis.

The Solar Array was assessed independently using a Biot-Savart model that analysed sensitivity to current ripple, string failure and solar array rotation angle. Harness contribution was analysed yielding a pT/A factor for DC and transient effects.
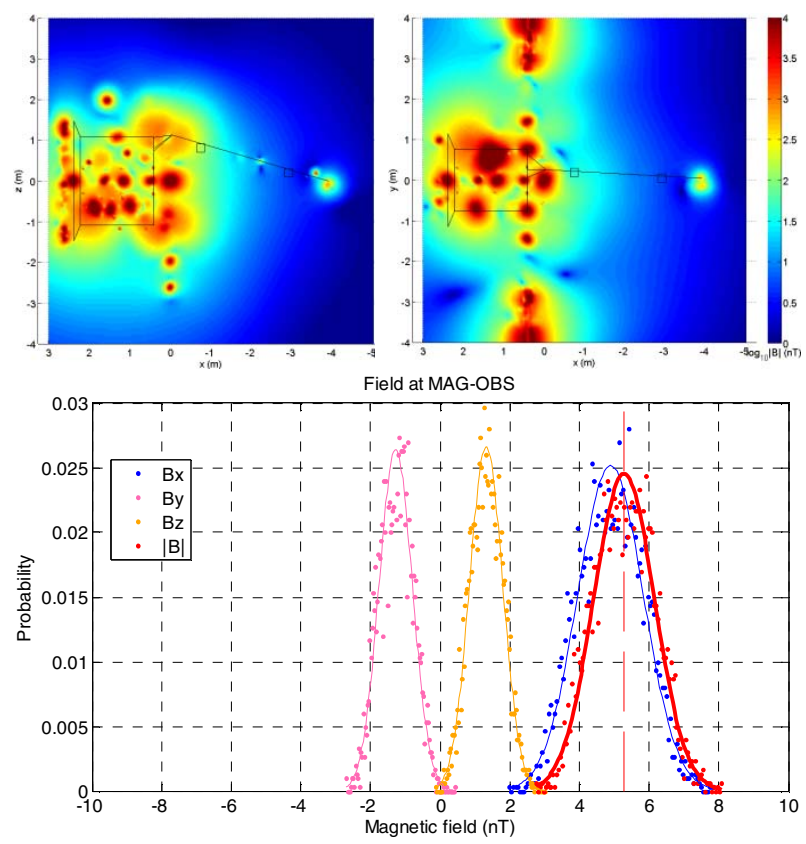

Figure 8. (top) Magnetic field distribution based on the magnetic budget model (bottom) Predicted field at MAG-OBS following a Monte Carlo simulation.

The DC magnetic budget has been analysed to predict a spacecraft field contribution at the MAG outboard sensor (MAG-OBS) of approximately 5nT, against a goal of $10 \mathrm{nT}$ and a requirement of $20 \mathrm{nT}$ (Fig. 8).

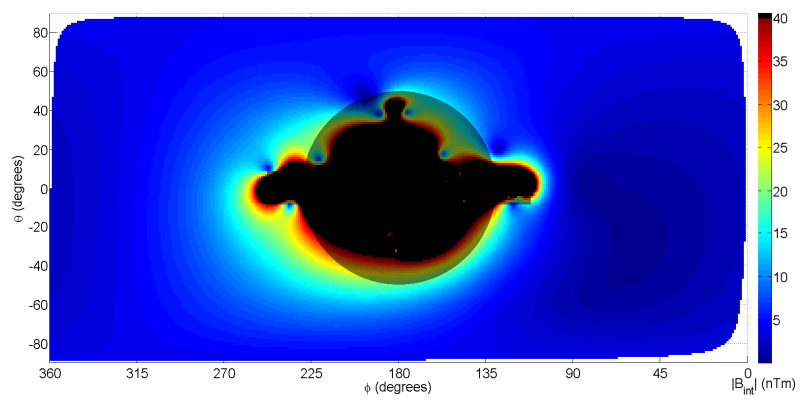

Figure 9. Integrated magnetic field, in angular space, as observed from the position of SWA-EAS on the end of the boom. The semi-transparent regions indicate a $50^{\circ}$ angle forming an agreed exclusion cone where distortion of particle trajectories due to the spacecraft was accepted to be outside the formal requirement during the design phase. 
The integrated magnetic field in the SWA-EAS field of view was also assessed, where the black regions in Fig. 9 indicate directions where the $40 \mathrm{nTm}$ specification is exceeded.

\subsection{Electric Field Analysis}

The DC electric field has been modelled by ESA and LESIA [6] to demonstrate compatibility with the SWAEAS and RPW-ANT instruments (Fig. 10). Note - some charging along the boom surface may occur due to the possible impact of predicted build-up of ice on the surface, which is caused by water from the bi-propellant thruster plume condensing onto the surface.

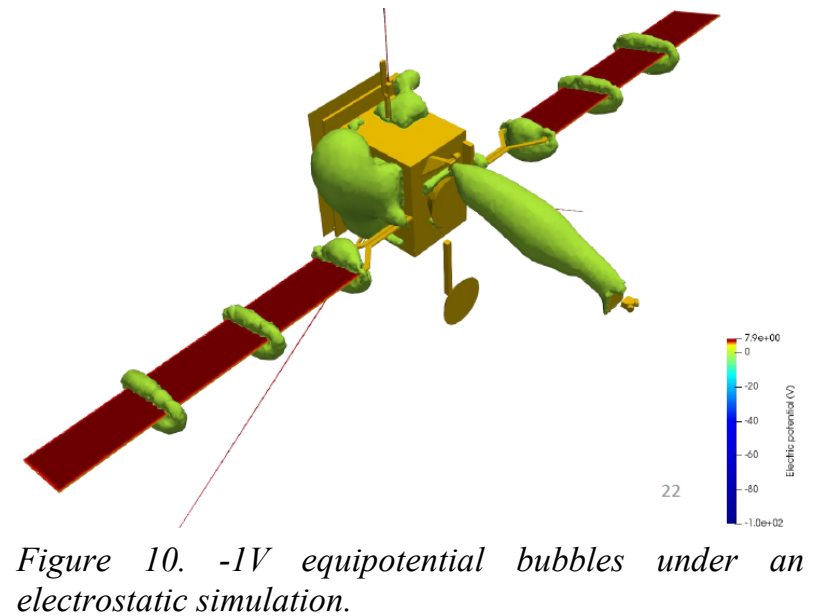

\subsection{AC Electric and Magnetic Field Analysis}

The AC electric and magnetic fields at system level have been modelled with predictions that will be checked during the EMC test campaign, and if necessary refined following testing to improve analysis against in-flight requirement levels.

In particular, the measurements made at close distance have to be scaled to the representative in-flight locations of the RPW-SCM and RPW-ANT instruments respectively. Where possible, the correct scaling law has been confirmed experimentally (e.g. for reaction wheel emissions, which were confirmed to fall-off with the expected cubic law from a measurement distance of $0.5 \mathrm{~m}$ ), otherwise a more conservative scaling has been applied (e.g. B $\alpha \mathrm{r}^{-2}$ until a distance greater than $3 \mathrm{x}$ the unit scale size has been reached, after which $\mathrm{B} \alpha \mathrm{r}^{-3}$ can safely be applied [7]).

Measurements of the shielding effectiveness of an equivalent sized honeycomb panel with aluminium face skins, for the low frequencies of interest, has also helped to de-risk the anticipated shielding effectiveness of the spacecraft structure. This demonstrated magnetic shielding due to eddy currents generated in the aluminium enclosure to be $11 \mathrm{~dB}$ at $1 \mathrm{kHz}$ up to $22 \mathrm{~dB}$ at $100 \mathrm{kHz}$, and for electric field shielding approximately
$27 \mathrm{~dB}$ from $10 \mathrm{kHz}$ to $20 \mathrm{MHz}$. These attenuating factors are included within the analysis to provide a prediction for the radiated magnetic and electric fields.

The results of this analysis have identified key frequencies and equipment to look out for during the RPW compatibility test in the EMC test campaign.

\section{SYSTEM LEVEL VERIFICATION}

EMC testing at spacecraft level is planned to take place towards the end of Q2, 2019. In addition to the standard compatibility tests with the launcher environment and auto-compatibility between the platform/payload with the communications subsystem, the campaign will include a compatibility test between the spacecraft and RPW suite of instruments inside of an anechoic chamber at IABG, Germany (IndustrieanlagenBetriebsgesellschaft $\mathrm{mbH}$ ).

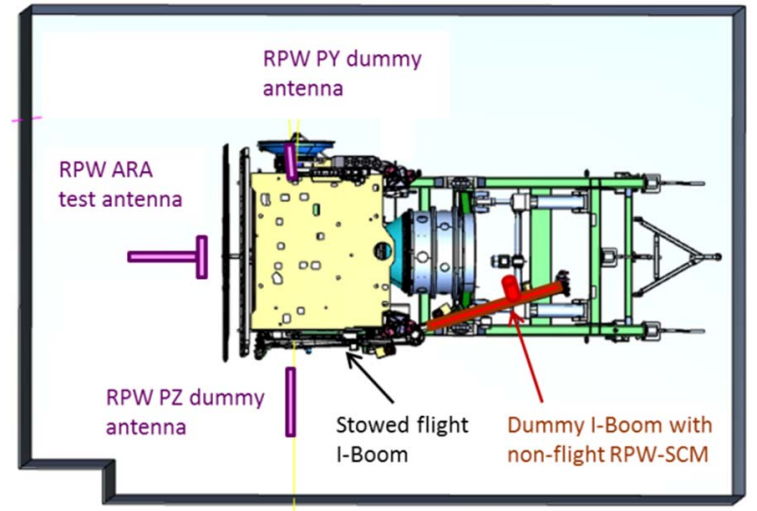

Figure 11. EMC RPW compatibility test set-up (additional IABG sensors not shown).

For this test, the flight appendages will remain stowed, and instead non-flight antennas will be mounted in representative in-flight locations at the RPW-SCM, $\mathrm{RPW}-\mathrm{ANT}+\mathrm{Z}$ and RPW-ANT $+\mathrm{Y}$ facing sides (PZ and $\mathrm{PY})$ to record the AC magnetic and electric fields. Independent field measurements (not connected to the S/C) will also be taken with a commercial ARA (Antenna Research Associates) antenna. The spacecraft will be configured with the heatshield facing horizontally, which allows access to the representative in-flight RPW-SCM location in the chamber (Fig. 11).

Additionally a full spacecraft magnetic test will be carried out at the IABG MFSA (Magnetic Field Simulation Facility) to measure the magnetic field generated at the MAG payload locations with the spacecraft both unpowered and powered. For these tests, an array of 10 sensors will be mounted, with 8 encircling the spacecraft approximately $1 \mathrm{~m}$ from the outer envelope, and 1 sensor at each of the MAG inflight representative locations, which necessitates the placement of sensors below the floor (Fig. 12). 
For the unpowered test the spacecraft, mounted on a non-magnetic trolley, will pass through the magnetometer sensor array in a series of steps from one side of the track to the other. The magnetic field at the in-flight MAG-OBS location will then be directly verified with sensor \#10, or independently extrapolated from measurements made at the other locations.
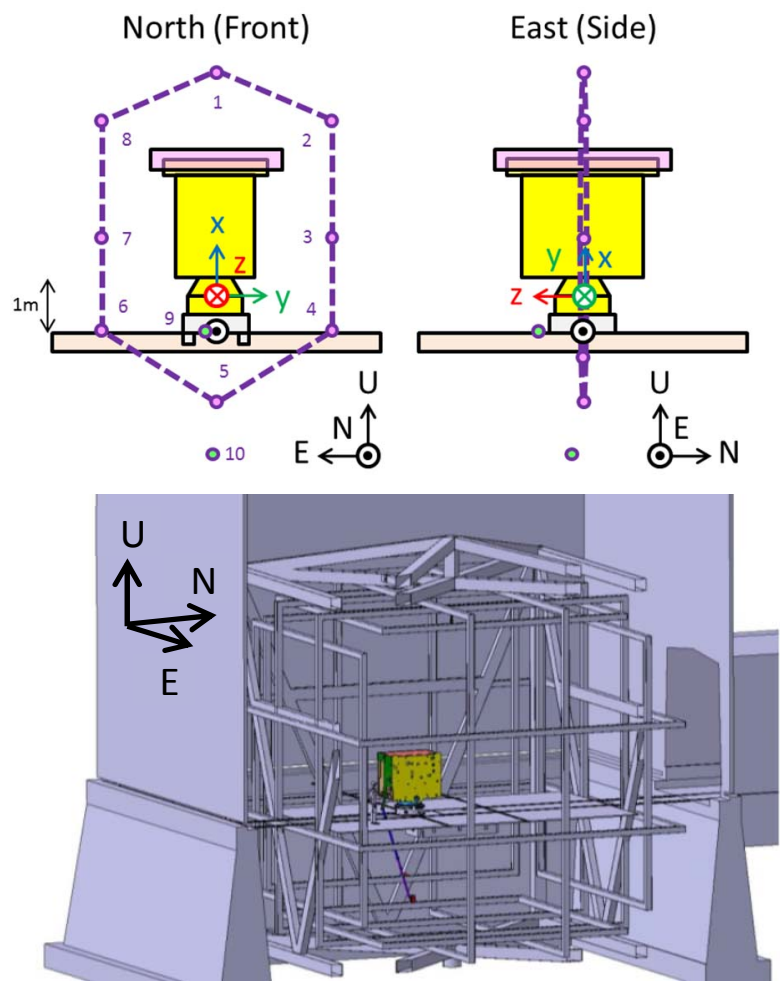

Figure 12. (top ) Sensor array around $S / C$ where $[x, y, z]$ indicate the $S / C$ co-ordinate system and [E,N,U] indicate the facility co-ordinate system (bottom) $S / C$ in Vertical orientation within the IABG MFSA.

For the powered measurement, the spacecraft will be positioned at the centre of the sensor array, and then switched on sequentially, allowing the perturbations from each power-up to be captured cumulatively. Once every relevant platform and instrument has been switched on into its scientific mode of operation, it will be possible to assess the powered contribution of the spacecraft to the DC magnetic field.

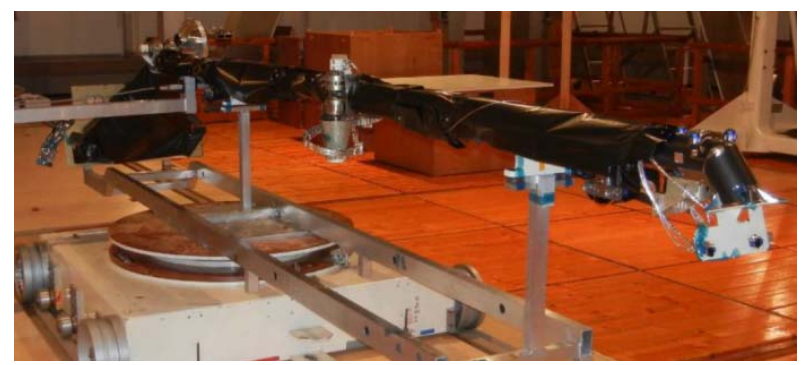

Figure 13. I-Boom installed on a non-magnetic trolley at IABG-MFSA.
The results from this test will then be superimposed on the independently measured I-Boom magnetic field contribution (already performed at IABG-MFSA, Fig. 13) to give a final verification of the field observed at MAG-OBS.

\section{CONCLUSION}

In summary, Solar Orbiter will take measurements of the inner heliosphere with an unprecedented synergy between in-situ and remote sensing instruments. To enable the state of the art payload to meet its scientific objectives, it has also pushed the boundaries of ESC/EMC control, analysis and test for such a system. The EMC WG was necessary and successful in establishing good engineering requirements, regularly meeting to communicate best practice on design, early measurements, and proposing solutions. This has allowed intervention where appropriate to ensure the necessary synergy between payloads (and platform) is maintained. The final verification of EMC requirements is planned to take place at IABG in Q2 2019.

Acknowledgements: The Solar Orbiter magnetometer was funded by the UK Space Agency under grant ST/P00217X/1.

\section{REFERENCES}

1. P. Narvaez, "The Magnetostatic Cleanliness Program for the Cassini Spacecraft," Space science reviews, 111(1):385-394, 2004.

2. A. Balogh, "Planetary Magnetic Field Measurements: Missions and Instrumentation," Space Science Review, (152):23-97, 2010.

3. A. Champlain. 2017. SPIS. [ONLINE] Available at: http://dev.spis.org/projects/spine/home/spis. [Accessed 26 September 2018].

4. ESA. 2018. Artist's impression of Solar Orbiter. http://sci.esa.int/solar-orbiter/55750-artist-simpression-of-solar-orbiter/. [Online: last updated 03/10/18, retrieved 23/01/19].

5. M. Burghoff, T. H. Sander, A. Schnabel, et al., "DC Magnetoencephalography: Direct measurement in a magnetically extremely-well shielded room." Applied Physics Letters, 85(25), 2004.

6. S. Guillemant, "Solar Orbiter / RPW and SWA EAS numerical simulations with the SPIS software", RPW-EAS-SYS-TN-001760-LES-MSSL, Issue 02 Rev 01, https://rpw.lesia.obspm.fr/ 18/04/2017,

7. M. Pudney, C. Carr, S. Schwartz et al., "Nearmagnetic-field scaling for verification of spacecraft equipment", Geoscientific Instrumentation, Methods and Data Systems, 2:249-255, 2013. 